\title{
Adaptación de las prácticas de Análisis Instrumental al Espacio de Convergencia Europeo. Elaboración de Material Didáctico
}

\author{
Ana Sayago, Dolores Hernanz, Valeria Gallo y Rafael Beltrán \\ Departamento de Química y Ciencia de los Materiales. Área de Química Analítica. Facultad de \\ ciencias Experimentales. Universidad de Huelva. Av. Tres de Marzo S/N. 21071. Huelva \\ (E-mail: ana.sayago@dqcm.uhu.es)
}

Recibido Dic. 15, 2010; Aceptado Ene. 20, 2011; Versión final recibida Ene. 30, 2011

\begin{abstract}
Resumen
En este trabajo se presenta el desarrollo de un proyecto de innovación educativa realizado por un equipo docente del Área de Química Analítica de la Universidad de Huelva en España como parte de la asignatura Análisis Instrumental de la carrera de Licenciatura en Química. Este proyecto intenta trasladar a los laboratorios un concepto más amplio de aprendizaje autónomo fomentando la capacidad del alumno a la hora de buscar, adquirir y elaborar los conocimientos, que se traduce en la elaboración de un protocolo de prácticas. El uso de este aprendizaje activo, que aumenta la motivación de los estudiantes, ha sido evaluado a través de una encuesta así como de entrevistas personales y tutorías grupales que han permitido conocer la satisfacción del alumno con la metodología utilizada.
\end{abstract}

Palabras clave: innovación docente, aprendizaje autónomo, laboratorios, protocolos de prácticas

\section{Adapting practices of Instrumental Analysis for European Convergence Space. Development of Training Materials}

\begin{abstract}
This paper presents the development of an educational innovation project done by the faculty team of the Department of Analytical Chemistry of the University of Huelva in Spain, as part of the course Instrumental Analysis of the major B.Sc. in Chemistry. This project tries to move to the laboratories a wider concept of self-learning, promoting the capacity of the students to search, acquire and develop knowledge, which they have to inform in protocol of practices report. The use of this active method of learning, which increases the motivation of the students, has been evaluated through a survey, through personal interviews and workshops that have allowed knowing the satisfaction of the students with the methodology used.
\end{abstract}

Keywords: teaching method innovation, self-learning, laboratories, lab protocols 


\section{INTRODUCCIÓN}

La unión económica, social, y política en Europa, ha supuesto lo que podría llamarse una recuperación de la idea universitaria del Renacimiento. La acción comunitaria en materia de educación, persigue decididamente el objetivo de promover la cooperación transnacional contribuyendo a crear un Espacio Europeo de Educación Superior (EEES; Declaración de Bolonia (1999). En las universidades, este espacio ha sido rediseñado adaptando sus estrategias de aprendizaje y enseñanza en los últimos años, con el objetivo de lograr una mejora en la calidad de los estudiantes, creando un entorno más interactivo y motivador tanto para alumnos como para profesores. Con este nuevo proceso, los estudiantes tendrán que dedicar más número de horas al trabajo personal y los profesores impartirán menos clases teóricas, sin embargo tendrán que diseñar nuevos métodos de aprendizaje con el fin de mejorar la enseñanza. (Drennan y Beck, 2001). Varios estudios presentados en la literatura internacional abordan metodologías modernas para ajustar planes, programas y métodos de enseñanza a este nuevo concepto del Espacio Europeo de Educación Superior y los aspectos pertinentes de la declaración de Bologna (Kirkwood-Tucker, 2004; Gavari, 2007; Ruiz et al., 2009; Murta y Armendáriz, 2010).

Frente al modelo pedagógico tradicional, que centra su desarrollo en la transmisión unidireccional de unos contenidos más o menos consolidados desde el profesor hacia el alumno, el modelo pedagógico que se propone desde la Convergencia Europea está focalizado en el estudiante y en su autonomía a la hora de buscar, adquirir y elaborar los conocimientos. En este último proceso de enseñanza-aprendizaje el profesor actúa como orientador o guía del alumno, ayudando a éste a descubrir aquello que quería transmitir, en vez de transferirlo sin más (Zabalza, 2002; De la Cruz, 2003). El aprendizaje autónomo dentro del marco de las actividades prácticas, parece una competencia consolidada si se tiene en cuenta que el alumno participa activamente en el desarrollo de las mismas. No obstante, la metodología didáctica más extendida entre el profesorado universitario en la enseñanza de asignaturas de carácter práctico es fundamentalmente unidireccional, en la que el alumno se limita básicamente a seguir las pautas indicadas en los protocolos de prácticas previamente elaborados por el profesor (Furió y col, 1999), ya que los contenidos de los mismos no se encuentran recogidos en uno o unos pocos textos de consulta, sino que aparecen dispersos en multitud de documentos de diversa índole.

La asignatura, Análisis Instrumental impartida en el tercer curso del programa licenciatura, presenta una parte teórica, que emplea fundamentalmente la lección magistral como herramienta metodológica, y una parte práctica realizada en el laboratorio, que permite aplicar y reforzar los conocimientos adquiridos durante las clases teóricas. En el proyecto de innovación docente que se discute en este trabajo se ha trasladado a los laboratorios el concepto de aprendizaje autónomo, fomentando una mayor implicación de los alumnos reforzando y ayudando a la comprensión de determinados contenidos propuestos en el programa de la asignatura. Además, el conocimiento del fundamento teórico mediante la búsqueda de información a partir de los recursos disponibles, favorece la motivación del alumnado que no se limitaría sólo a seguir los protocolos. Esta motivación constituye uno de los aspectos determinantes en el proceso de aprendizaje. El alumno motivado se concentra más en lo que está haciendo, es más persistente y dedica más tiempo y esfuerzo. Por otro lado, el alumno se ha familiarizado con el uso de nuevos recursos y herramientas imprescindibles en su futuro profesional.

En este trabajo se presenta el desarrollo de un proyecto de innovación docente realizado por un equipo docente del Área de Química Analítica de la Universidad de Huelva dentro del marco de la asignatura Análisis Instrumental de la licenciatura de Química. Este proyecto intenta trasladar a los laboratorios un concepto más amplio de aprendizaje autónomo fomentando la capacidad del alumno a la hora de buscar, adquirir y elaborar los conocimientos, que se traduce en la elaboración de un protocolo de prácticas.

\section{METODOLOGÍA}

Para el desarrollo del proyecto se plantearon dos objetivos generales. Como primer objetivo se buscó implicar directamente al alumnado en el aprendizaje práctico de la asignatura análisis 
instrumental, concretamente en la elaboración de material didáctico que además de ser utilizado como herramienta para su propia formación servirá como material de apoyo a cursos posteriores. El segundo objetivo resultó ser un compendio de objetivos formativos generales tales como: desarrollar el aprendizaje autónomo, potenciar la creatividad, favorecer la capacidad crítica y autocrítica, tratar adecuadamente el extenso volumen de información existente en diversas fuentes y recursos bibliográficos y por último fomentar la autonomía y libertad responsables y, al mismo tiempo, el trabajo en equipo, favoreciendo el desarrollo de habilidades de interacción humana.

Para la consecución de estos objetivos se puso en marcha una serie de actividades perfectamente estructuradas en el espacio y en el tiempo. La figura 1 muestra un esquema de la metodología seguida. En primer lugar, el equipo docente se reunió al inicio del curso académico para seleccionar las prácticas que se iban a realizar. Asimismo, se debatieron todos los aspectos de coordinación de contenidos y diseño de las actividades necesarias para este fin.

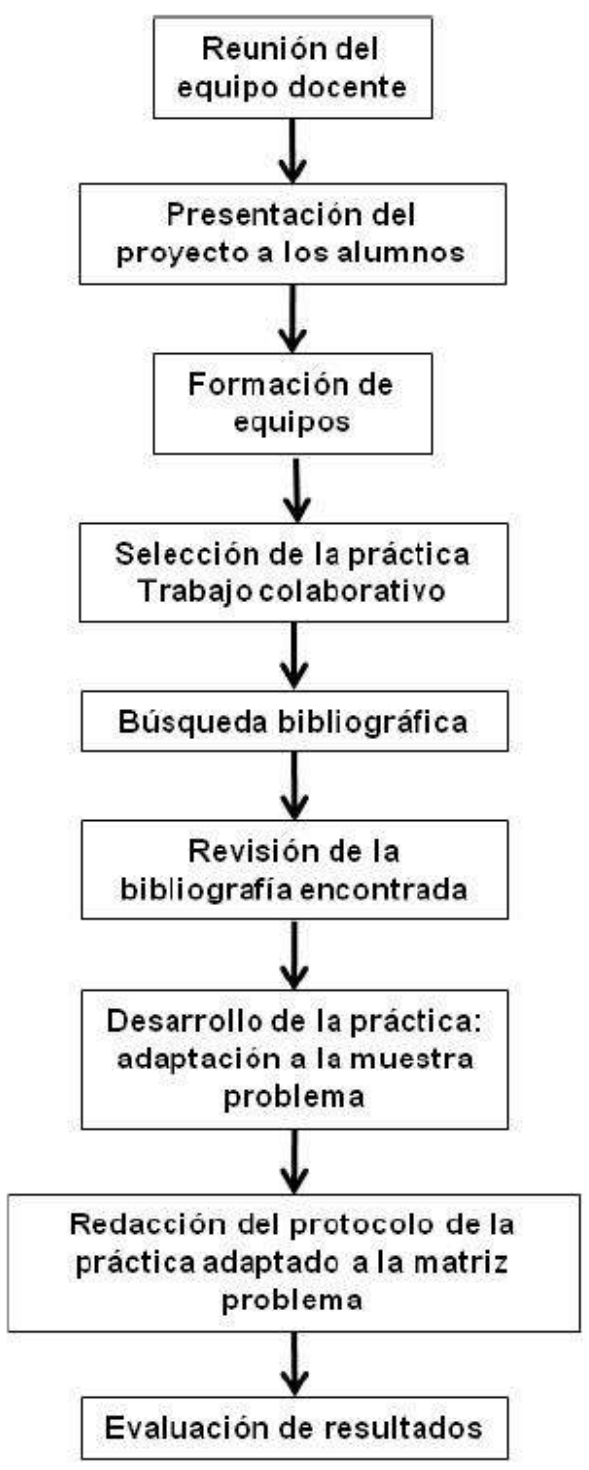

Fig. 1: Esquema de la metodología seguida para el desarrollo del proyecto

Posteriormente, se presentó el proyecto a los alumnos. Se instó a los alumnos a participar en éste, considerándolo como un componente más para la evaluación, favoreciendo de este modo un mayor grado de compromiso. Se formaron grupos de trabajo a los que se les dio a elegir la práctica que debían desarrollar, y se les enseño la utilización de los distintos recursos bibliográficos disponibles en la Universidad de Huelva (biblioteca, bases de datos, etc.). 
Durante la primera sesión práctica, se revisó el material bibliográfico encontrado por el alumno y se seleccionó el más adecuado para la resolución del problema analítico. En las sesiones prácticas que siguieron, se adaptaron los protocolos de análisis a las muestras problema objeto de estudio. Tras la resolución, cada grupo de trabajo, elaboró su protocolo de prácticas y se evaluaron los resultados a través de una encuesta así como de entrevistas personales y tutorías grupales.

\section{RESULTADOS Y DISCUSIÓN}

\section{Grado de cumplimiento de los objetivos}

En relación al primer objetivo fijado, el alumnado se ha sentido completamente implicado en el aprendizaje práctico de la asignatura y ha sido capaz de elaborar el protocolo correspondiente a la práctica seleccionada.

Asimismo, el grado de cumplimiento relacionado con el desarrollo de los objetivos formativos generales que fueron planteados como segundo gran objetivo de este proyecto, podría concretarse de la siguiente manera:

i) Desarrollo del aprendizaje autónomo: el desarrollo del proyecto ha permitido que el alumno investigue empleando las distintas fuentes de información que se encuentran a su alcance, favoreciendo de esta forma que se familiarice con el uso de recursos y herramientas imprescindibles en su futuro profesional.

ii) Potenciar la creatividad: la necesidad de redactar un texto ilustrado de fácil comprensión para sus futuros compañeros, ha favorecido la puesta en marcha de su imaginación e ingenio haciendo posible la consecución del protocolo.

iii) Favorecer la capacidad crítica y autocrítica: seleccionando entre toda la información aquella más conveniente para la realización de las prácticas. Por otra parte han evaluado el trabajo de todos los miembros del equipo, así como el suyo propio.

iv) Tratar adecuadamente el extenso volumen de información existente en diversas fuentes y recursos bibliográficos: este objetivo se desarrolló de forma conjunta al anterior, ya que la capacidad de crítica es necesaria para extraer de toda la información recopilada, aquella que resulta fundamental.

v) Fomentar la autonomía y libertad responsables y, al mismo tiempo, el trabajo en equipo, favoreciendo el desarrollo de habilidades de interacción humana: Se ha querido fomentar el trabajo en grupo como una herramienta eficaz en la resolución de tareas, de forma que sea tan importante una solución acertada, como el debate que se genera entre alumnos. Estos, pueden tener más éxito que el propio profesor para hacer entender ciertos conceptos a sus compañeros (aprendizaje horizontal, de igual a igual).

\section{Elaboración de material didáctico.}

Siguiendo los pasos establecidos por la metodología, el alumno tuvo que buscar en las diferentes fuentes bibliográficas, información relacionada con el problema analítico que debían resolver durante la semana de prácticas. Esta información fue revisada por el equipo docente, que actuó como guía ayudando al alumno a seleccionar aquella que resultase más adecuada para el desarrollo de la práctica. En las sesiones de prácticas que siguieron, se desarrollaron las experiencias necesarias adaptando la información encontrada a las matrices objeto del problema en estudio. Una vez resuelto el problema analítico, los alumnos trabajaron en equipo para elaborar el protocolo de laboratorio. Se establecieron horarios de tutoría para la consulta de aquellos aspectos que pudiesen requerir de la opinión del profesor y a los quince días se realizó una tutoría grupal con todos los estudiantes con el fin de aclarar aquellos aspectos más demandados por los mismos. Como se esperaba, durante las tutorías realizadas con los diferentes grupos, se observaron deficiencias generales en la confección de los protocolos, por lo que, en la primera sesión general además de aclarar los aspectos solicitados, los profesores presentaron un guión 
orientador que fue distribuido a todos los estudiantes. Este material surtió el efecto esperado, pues los protocolos presentados en las subsiguientes tutorías de equipo resultaron ser de mejor calidad y con un formato uniforme.

\section{Evaluación de los resultados del proyecto.}

Como principal resultado de este proyecto, podría destacarse el elevado grado de satisfacción que han mostrado los alumnos con el desarrollo de éste a lo largo de las sesiones presenciales, así como en su "labor investigadora". Esto ha sido contrastado con entrevistas personales y grupales, así como con cuestionarios a través de la plataforma de enseñanza virtual de la Universidad (Moodle). La figura 2 muestra las preguntas planteadas a los alumnos así como la valoración promedio obtenida para las mismas. Cada ítem fue valorado en una escala de uno a cuatro.

\section{CUESTIONARIO}

1. Los objetivos del proyecto han sido definidos claramente por el profesor.

2. Mi interés por el tema de la práctica ha ido aumentando a lo largo de su desarrollo.

3. El tamaño del grupo permitió el aprovechamiento de la actividad.

4. El ambiente de trabajo ha sido agradable.

5. La actividad favorece el desarrollo de mi capacidad para trabajar en equipo.

6. La actividad favorece la aplicación de conocimientos.

7. La actividad favorece el desarrollo mi sentido crítico.

8. La actividad propuesta favorece el aprendizaje autónomo.

9. Mis compañeros me han ayudado a resolver mis dudas.

10.La actividad ha ayudado a la comprensión de la materia.

11. He sentido que el trabajo entregado me pertenecía.

12. Todos los compañeros se han entregado en la elaboración del trabajo.

13.El tutor nos facilita los recursos básicos para indagar sobre el tema.

14.En general, estoy satisfecho con el trabajo desarrollado.

15.En general, estoy satisfecho con el trabajo llevado a cabo por el profesor.

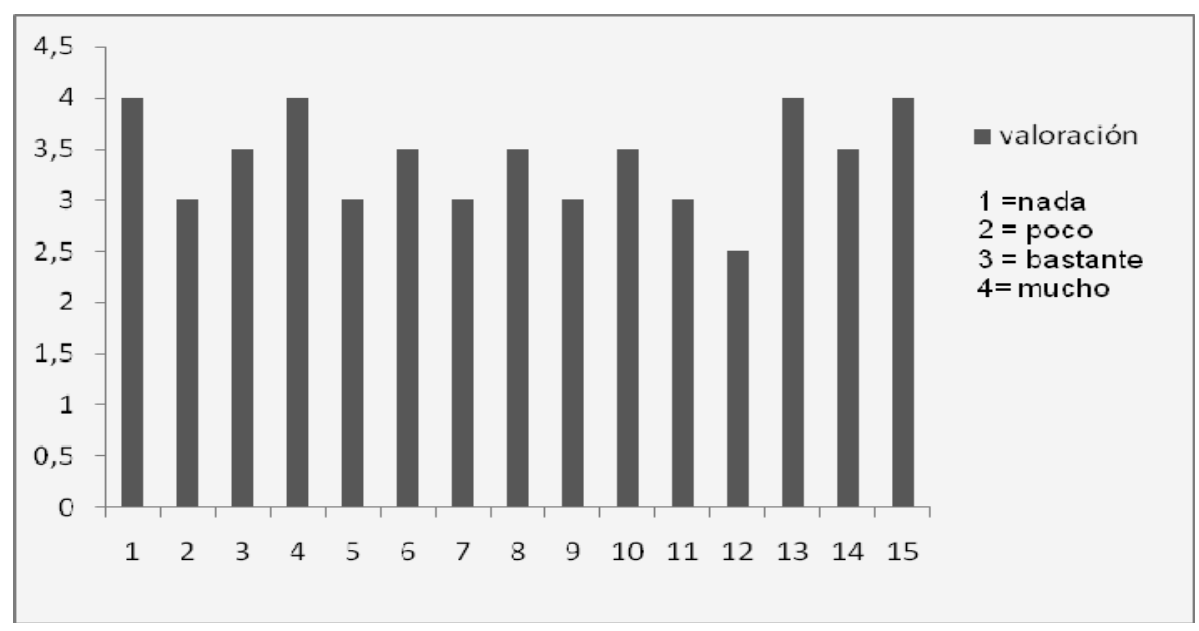

Fig. 2: Cuestionario realizado a través de la plataforma de enseñanza virtual y análisis de los resultados.

Mediante esta nueva experiencia de innovación docente llevada a cabo en el ámbito práctico de la asignatura de Análisis Instrumental, el alumno ha sido capaz de construir su conocimiento de manera personal pero mediada por la intervención del profesor o de otros alumnos mejor preparados. Esta idea es en definitiva lo que persigue la implantación del ECTS en el EEES, "aprender a aprender". La actividad conjunta de profesores y alumnos, durante las tutorías grupales y el trabajo en equipo ha permitido este proceso de construcción del conocimiento en un ambiente agradable y distendido, que ha favorecido un desarrollo dinámico y atractivo de las 
clases prácticas. El trato y convivencia durante las jornadas previas y posteriores a las prácticas así como las cinco horas diarias a lo largo de una semana con los alumnos, ha facilitado el conocimiento de los mismos por parte de los profesores, y ha permitido una atención individualizada de las necesidades que se planteaban en cada momento.

\section{CONCLUSIONES}

El desarrollo de este proyecto ha supuesto un reto importante para los profesores implicados, ya que el trabajo dentro del laboratorio requiere una atención y supervisión personalizada, en todo momento sobre la práctica llevada a cabo por el alumno. A pesar de esto, ha resultado una experiencia satisfactoria ya que los objetivos han sido alcanzados en su inmensa mayoría. Además, ha permitido al alumno relacionar los contenidos adquiridos durante las sesiones presenciales de teoría con los trabajados durante las sesiones prácticas en el laboratorio.

El trabajo en grupo ha sido de gran riqueza formativa; ha constituido una buena ocasión para completar mutuamente la formación del profesor y los alumnos y ha supuesto además para el alumno, un acercamiento al conocimiento y manejo de referencias bibliográficas analíticas e instrumentales, que les serán de extraordinaria ayuda a la finalización de sus estudios. Este tipo de estrategia docente debería aplicarse en un futuro a las diferentes disciplinas de carácter práctico en las carreras de ciencias, ya que el alumno aprende a desenvolverse en el laboratorio para resolver un problema, alejándose mucho de la forma tradicional de "elaborar recetas" a la que suelen estar acostumbrados.

\section{AGRADECIMIENTOS}

Los miembros del equipo docente agradecen al servicio de Innovación Docente de la Universidad de Huelva, por la oportunidad que les ha brindado al concederles este proyecto.

\section{REFERENCIAS}

De la Cruz, M.A., El proceso de Convergencia Europea: ocasión de modernizar la universidad española si se produce un cambio de mentalidad en gestores, profesores y estudiantes. Aula Abierta, 82, 191-216 (2003)

EEES, Declaración de Bolonia. Declaración conjunta de los ministros europeos de educación reunidos en Bolonia el 19 de junio de 1999, www.coitm.org/Impresos/Bolonia/3Declaracion.pdf, Bolonia (1999)

Drennan, L.T. y M. Beck, Teaching quality performance indicators - key influences on the UK universities'scores. Quality Assurance Education: 9(2), 92-102 (2001)

Furió, C., Gil, D. y Valdés, P., ¿Tiene sentido seguir distinguiendo entre aprendizaje de conceptos, resolución de problemas de lápiz y papel y realización de prácticas de laboratorio?. Enseñanza de las ciencias: revista de investigación y experiencias didácticas, 17(2), 311-321 (1999)

Gavari, E., The Practicum: An Example Of Changes In The Teaching And Learning Process In The European Higher Education Space, Odgojne znanosti 9(1), 119-134 (2007)

Kirkwood-Tucker, T.F., Toward a European Model of Higher Education: Processes, Problems, and Promises, European Education, 36(3), 51 - 69 (2004)

Murta, P.M. y Armendáriz, J.E., On the Utility of ICT in the European Higher Education Area: The Bologna Process and Its Implications in the Innovation of the Teaching and Learning Process, Communications in Computer and Information Science, 73, 216-222, (2010)

Ruiz M., Tirado P. y Menor E., On our way to the European higher education space. A proposal for a teaching guide for the management control course in the business management and administration degree, US-China Education Review, 6(6), 9-23 (2009)

Zabalza, M.A. La enseñanza Universitaria: El escenario y sus protagonistas, In El alumnado universitario, pág. 107-131 Ed. Narcea, SA. Ediciones, Madrid, España (2002) 